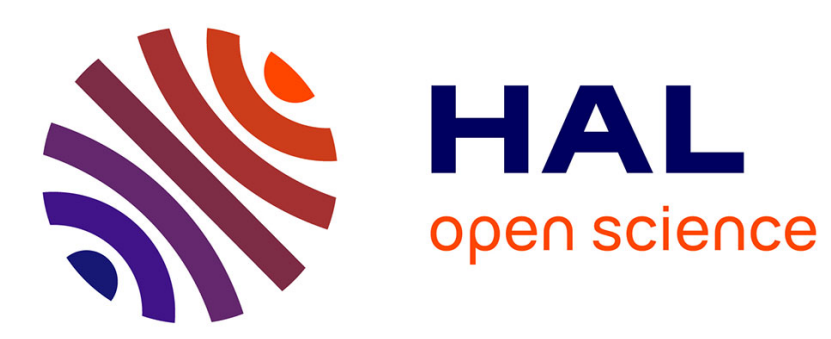

\title{
Constructive root bound for k-ary rational input numbers
}

Sylvain Pion, Chee Yap

\section{To cite this version:}

Sylvain Pion, Chee Yap. Constructive root bound for k-ary rational input numbers. Theoretical Computer Science, 2006, 369 (1-3), pp.361-376. 10.1016/j.tcs.2006.09.010 . inria-00344349

\section{HAL Id: inria-00344349 \\ https://hal.inria.fr/inria-00344349}

Submitted on 4 Dec 2008

HAL is a multi-disciplinary open access archive for the deposit and dissemination of scientific research documents, whether they are published or not. The documents may come from teaching and research institutions in France or abroad, or from public or private research centers.
L'archive ouverte pluridisciplinaire HAL, est destinée au dépôt et à la diffusion de documents scientifiques de niveau recherche, publiés ou non, émanant des établissements d'enseignement et de recherche français ou étrangers, des laboratoires publics ou privés. 


\title{
Constructive Root Bound for $k$-Ary Rational Input Numbers
}

\author{
Sylvain Pion ${ }^{1}$ \\ INRIA Sophia-Antipolis, \\ BP 93, 06902 Sophia-Antipolis cedex, France. \\ Chee K. Yap ${ }^{1}$ \\ Courant Institute of Mathematical Sciences, New York University \\ New York, NY 10012, USA
}

\begin{abstract}
Guaranteeing accuracy is the critical capability in Exact Geometric Computation, an important paradigm for constructing robust geometric algorithms. Constructive root bounds is the fundamental technique needed to achieve such guaranteed accuracy. Current bounds are overly pessimistic in the presence of general rational input numbers. In this paper, we introduce a method which greatly improves the known bounds for $k$-ary rational input numbers. Since a majority of input numbers in scientific and engineering applications are either binary $(k=2)$ or decimal $(k=10)$, our results could lead to a significant speedup for a large class of applications. We apply our method to two of the best available constructive root bounds, the BFMSS Bound and the Degree-Measure Bound. Implementation and experimental results based on the Core Library are reported.
\end{abstract}

Key words: Constructive root bounds, exact geometric computation, robust numerical algorithms, k-ary rational numbers

Email addresses: Sylvain.Pion@sophia.inria.fr (Sylvain Pion), yap@cs.nyu.edu (Chee K. Yap).

1 This research is supported by NSF/ITR Grant \#CCR-0082056. Sylvain's work is conducted under a postdoc fellowship with this grant. 


\section{Introduction}

The critical idea of the Exact Geometric Computation (EGC) approach to robust geometric algorithms is "geometric exactness". This amounts to ensuring that all computational decisions in a program are error free. It translates to the ability to guarantee the sign of real numerical quantities. Guaranteeing the sign is a special form of "guaranteed accuracy computation" [16]. In guaranteed accuracy computation, we can pre-specify an accuracy for each numerical quantity. Guaranteeing the sign of a number amounts to guaranteeing one relative bit of the number. Such techniques have been encoded into two general libraries LEDA_real $[6,1]$ and Core Library [4,5]. To ensure this form of numerical control, the use of root bounds is central.

To illustrate this idea, suppose $\alpha$ is an algebraic number that is given via some expression $E$, involving constants and numerical operations. Now, suppose we have some method for computing a number $\beta(E)$ with the property that if the value of $E$ (which is $\alpha$ ) is non-zero then $|\alpha| \geq \beta(E)$. Such a number $\beta(E)$ is called a root bound for $E$ (or, for $\alpha$ ) in this paper. For example, let $\alpha_{0}$ be

the value of the expression $E_{0}=\sqrt{2}+\sqrt{3}-\sqrt{5+2 \sqrt{6}}$. It is known that we can choose $\beta\left(E_{0}\right)=2^{-54}$ for our root bound (see Table 1 below). Now, if we approximate $\alpha_{0}$ to at least 55 bits of absolute accuracy, and discover that the approximate $\alpha_{0}$ is less than $2^{-55}$, we can conclude that $\alpha_{0}$ is in fact 0 . On a typical hand calculator, we carry out such an approximation of $\alpha_{0}$ and obtain the approximate value $6.3376 \times 10^{-38}$. So $\alpha_{0}$ must be 0 .

Now there are many known classical root bounds (e.g., [9]), but these are usually non-constructive in the sense that it depends on parameters that cannot be easily deduced from the expression $E$. What we need are called constructive root bounds in [8]. Such bounds are defined relative to some set $\mathcal{E}$ of algebraic expressions. It is constructive in two ways: (i) First, for each expression $E \in \mathcal{E}$, we define a set of mutually recursive parameters $u_{1}(E), \ldots, u_{m}(E)$ (ii) Second, there is an explicit computable root bound function $\beta\left(u_{1}, \ldots, u_{m}\right)$ such that if $E$ is well-defined and $E \neq 0$, then

$$
|E| \geq \beta\left(u_{1}(E), \ldots, u_{m}(E)\right) .
$$

We will write $\beta(E)$ instead of $\beta\left(u_{1}(E), \ldots, u_{m}(E)\right)$. To be more precise, we may call $\beta$ an exclusion root bound; if the inequality in (1) were reversed, we would have an inclusion root bound.

The first example of such constructive root bounds is Mignotte's constructive Measure Bound [10], applied to the problem of "identifying algebraic numbers". The measure bound has been sharpened by Sekigawa [14]. In EGC, such bounds were first introduced in the Real/Expr Package [17], where the 
degree-height bounds [17] and degree-length bounds [15, p. 177] were used. Scheinerman [12] gave a constructive bound for algebraic integers based on eigenvalues. Burnikel et al. [2] introduced the BFMS Bound that turns out to be extremely effective for division-free expressions. Recently, this bound was improved to what we will call ${ }^{2}$ the BFMSS Bound [3]. In [8,7], we introduced another constructive root bound that overcomes some of the shortcomings of BFMS. If $\beta, \beta^{\prime}$ are root bound functions, we can compare them in two ways: (i) efficiency and (ii) effectiveness. Efficiency refers to the complexity of computing the root bounds, and effectivity refers to the size of the bounds (a larger $\beta(E)$ is more effective). Generally, the most interesting comparison is based on effectiveness (efficiency is less of an issue in most applications because the running time is usually dominated by the multiprecision arithmetic). If $\beta^{\prime}(E) \geq \beta(E)$ for all $E \in \mathcal{E}$, we say $\beta^{\prime}$ dominates $\beta$ (over $\mathcal{E}$ ). Among the current constructive root bounds, there are three that are not dominated by any others over the class of constructible expressions: degree-measure [10,2], BFMSS [3] and Li-Yap [8]. We give a comparison of the effectiveness of these three root bounds in Section 6.

The starting point of this paper is the observation that (a) current constructive bounds are quite effective for division-free input expressions involving only integer inputs, and (b) the bounds become considerably worse in the presence of division. Even when the expression is division-free, the presence of rational input numbers counts as introducing division into the expression. Such ineffective bounds can make some computations impractical. We note that these ineffective bounds are sometimes intrinsic, because it is easy to see that the worst case requires exponential bit sizes. Fortunately, this is not the end of the story. The vast majority of numerical input in scientific and engineering applications involves $k$-ary rationals for some integer $k \geq 2$. Invariably $k=2$ (binary) or $k=10$ (decimal). By a $k$-ary rational we mean a rational number whose denominator is a power of $k$. Thus $k$-ary rationals are generalizations of integers.

We shall introduce a general technique that can take advantage of $k$-ary rationals. The technique seems orthogonal to previous techniques in the sense that for any current constructive root bound $\beta$, we can modify it to a " $k$-ary version" $\beta_{k}$ which is more effective. In this paper, we introduce the $k$-ary version of the BFMSS and Measure Bounds. These will be referred to as the BFMSS $[k]$ and Measure $[k]$ Bounds. In algorithms, especially in computer algebra, it is a well-known phenomenon that rational number arithmetic is much slower than integer arithmetic. However, $k$-ary rational number arithmetic has a complexity that is intermediate between these two extremes. The techniques of this paper will yield the same kind of intermediate complexity for root bounds of expressions with $k$-ary input numbers.

$\overline{2}$ The BFMS and BFMSS bounds are both named after the initials of their authors. 
Some Examples. We briefly illustrate the possible improvements with our new technique. Instead of the root bound $\beta(E)$, we usually consider the corresponding bit-bound, defined as $-\lg \beta(E)$.

An example from [8] is the identically zero expression $E_{1}(x, y)=\sqrt{x}+\sqrt{y}-$ $\sqrt{x+y+2 \sqrt{x y}}$. Suppose $x, y$ are $L$-bit binary numbers (i.e., numerators are $L$-bit integers and denominators are $L$-bit powers of 2 ). Table 1 compares some bit-bounds and timings (cf. [3]). Line 1 gives the bit-bound as a function of $L$. Line 2 gives the range of bit-bounds computed by our Core Library implementation when 10 random choices of double precision floating-point machine numbers are substituted for $x$ and $y$. Line 3 gives the time to evaluate the 10 random examples of Line 2 for 100 times each.

Table 1

Comparison of BFMSS, Li-Yap and BFMSS[2]

\begin{tabular}{|l|l||l|l|l|}
\hline & Method & BFMSS & Li-Yap & BFMSS [2] \\
\hline \hline 1 & Bit-Bound function & $96 L+30$ & $28 L+60$ & $8 L+30$ \\
\hline 2 & Bit-Bound Range $(L=53)$ & $4926-5118$ & $2085-2165$ & $426-462$ \\
\hline 3 & Timing $(L=53,100 \times 10$ times $)$ & $46.7 \mathrm{~s}$ & $8.35 \mathrm{~s}$ & $3.58 \mathrm{~s}$ \\
\hline
\end{tabular}

When $x, y$ are rational numbers whose numerators and denominators are $L$-bit integers, the Bit-Bound functions for BFMSS and Li-Yap are just $96 L+30$ and $28 L+60$ (as in Line 1) while BFMSS[2] drops to $8 L+30$. On the other hand, when $x, y$ are $L$-bit integers, the Bit-Bound function for all three methods is the same and equal to $7.5 L+30$. This example illustrates our previous remark, that our new bit-bounds for $k$-ary input numbers lie between the bit-bounds for integers and for rational numbers. Indeed, they are only slightly worse than the integer case.

Next, consider the important and common situation of evaluating $n \times n$ determinants where the input numbers are $L$-bit binary numbers. Such numbers have the form $m 2^{-k}$ where $|m|<2^{L}$ and $0 \leq k \leq L$. Let $E_{0}$ be an expression for such a determinant. First, assume $E_{0}$ is the co-factor expansion of the determinant (this is a polynomial with $n$ ! terms). Then the BFMSS Bound for $E_{0}$ gives a root-bit bound that is more than

$$
(n !) n L .
$$

This is exponentially worse in $n$ than our binary version of the BFMSS Bound, which gives a root-bit bound of $2 n L$.

In our experiments (Section 6), we use a more efficient determinant expression: let $E_{1}$ be the determinant expression obtained by using dynamic programming principles. Thus $E_{1}$ is a DAG while $E_{0}$ is a tree. E.g., when the input is a 
random $5 \times 5$ matrix and $L=100$, our BFMSS implementation gives the bound $-\lg |E| \geq 10,282$, while our binary version of BFMSS gives $-\lg |E| \geq 326$.

Overview. Section 2 gives a high-level view of what our $k$-ary transformation does to any constructive root bound. Section 3 reviews the BFMSS Bound, while Section 4 gives the new BFMSS[k] Bound. We show that BFMSS[k] dominates BFMSS. Section 5 gives the new Measure $[\mathrm{k}]$ Bound, and again we show that Measure $[\mathrm{k}]$ dominates Measure. Experiments and comparisons are given in Section 6. We conclude in Section 7.

\section{Generic k-Ary Method}

We propose a meta-method for exploiting $k$-ary input numbers. The metamethod is applicable to any constructive root bounding method. In particular, we will apply it to the BFMSS Bound and the Measure Bound. In general, if $\beta$ is a root bound function as in (1), our $k$-ary transformation produces a related root bound function $\beta_{k}$. Writing $\beta^{\text {bfmss }}$ and $\beta^{\text {meas }}$ for the root bound functions corresponding to the BFMSS and Measure Bounds, we will describe their $k$-ary versions, $\beta_{k}^{\text {bfmss }}$ and $\beta_{k}^{\text {meas }}$.

As usual, we consider the class of expressions which are DAGs with ${ }^{3}$ rational numbers at the leaves and whose internal nodes are algebraic operators. The typical class of algebraic operators are,,$+- \times, \div$ and algebraic root extraction, but this may vary depending on context. Let $\operatorname{val}(E)$ be the algebraic number denoted by $E$. Since algebraic operators are partial functions, $\operatorname{val}(E)$ may be undefined. In any inequality involving val $(E)$, it is understood that the inequality is in effect only when both sides are defined. We usually write " $E$ " instead of $\operatorname{val}(E)$ when this is clear from context.

The basic idea of the $k$-ary transformation is to transform an expression $E$ to another expression $E_{k}$, such that $E$ and $E_{k}$ are connected by

$$
E=k^{v_{k}(E)} E_{k}
$$

for some $v_{k}(E) \in \mathbb{Z}$. What are the constraints on this transformation? If $\beta(E)$ is the original root bound function, this transformation will lead naturally to

\footnotetext{
3 In previous papers on constructive root bounds, leaves of expressions are assumed to be integers (e.g.. Table 2). This is because rational numbers can be simulated by a division step. In the present paper, we allow $k$-ary rationals at the leaves in order to avoid introducing a general division. But since $k$ may vary, we simply admit all rational numbers in this discussion.
} 
a corresponding $k$-ary root bound $\beta_{k}(E)$. In this paper, our basic goal is to ensure that $\beta_{k}$ dominates $\beta$ :

$$
\beta_{k}(E) \geq \beta(E)
$$

for $E \in \mathcal{E}$. Achieving this inequality will depend on the nature of $\beta$. Assuming both sides of (3) are well-defined, we have

$$
\begin{aligned}
E \neq 0 & \Rightarrow E_{k} \neq 0 \\
& \Rightarrow\left|E_{k}\right|>\beta\left(E_{k}\right) \\
& \Rightarrow|E|>k^{v(E)} \beta\left(E_{k}\right) .
\end{aligned}
$$

Thus we define

$$
\beta_{k}(E):=k^{v(E)} \beta\left(E_{k}\right)
$$

and so the inequality (4) amounts to $\beta\left(k^{v(E)} E_{k}\right) \leq k^{v(E)} \beta\left(E_{k}\right)$.

To simplify ${ }^{4}$ the presentation below, we will choose $k=2$. Also, we will simply write $v(E)$ instead of $v_{2}(E)$. Generalizing this to a general $k>2$ is mostly straightforward. A further generalization is to maintain the powers of two or more $k$ 's simultaneously. It seems that $\left(k^{\prime}, k^{\prime \prime}\right)=(2,5)$ will yield most of the benefits of the method, since actual input numbers in computation are overwhelmingly decimal or binary. This amounts to the following transformation (cf. (3)):

$$
E=2^{v_{2}(E)} 5^{v_{5}(E)} E_{2,5}
$$

where $v_{k}(E) \in \mathbb{Z}($ for $k=2,5)$.

\section{The BFMSS Bound}

We first review the BFMSS Bound [2,3] for algebraic expressions. Let $E$ be an expression as represented by a DAG, with integers at its leaves, and whose internal nodes correspond to the operators in column 1 of Table 2. The "diamond operator" in the last row of the Table extracts the $j$ th largest real root of the polynomial $\sum_{i=0}^{n} F_{i} X^{i}$ where $F_{i}$ are expressions. For this instance of the diamond operator, we associate an inclusion root bound function (in the sense of $[3])$,

$$
\Phi\left(a_{n-1}, \ldots, a_{i}, \ldots, a_{0}\right)
$$

$\overline{4}$ The case $k=2$ is the most important case. Also, the resulting formulas are easier to read as we avoid the use of the variable $k$. 
where each $a_{i}$ is to be replaced by $F_{i} / F_{n}$. We will simply write " $\Phi\left(\ldots, a_{i}, \ldots\right)$ " instead of (6) where it is understood that the index $i$ decreases from $n-1$ to 0 . In other words, $\Phi\left(\ldots, a_{i}, \ldots\right)$ is an upper (i.e., inclusion) bound on all real roots of the polynomial $X^{n}+a_{n-1} X^{n-1}+\cdots+a_{0}$. Since there are several possible choices $\Phi_{1}, \Phi_{2}$, etc for $\Phi$, we may just compute the bound given by each $\Phi_{i}$ and take the best. This procedure amounts to the observation that if $\Phi_{1}$ and $\Phi_{2}$ are inclusion root bound functions, then $\min \left\{\Phi_{1}, \Phi_{2}\right\}$ is also an inclusion root bound.

Table 2

BFMSS Rules

\begin{tabular}{|c||c|c|}
\hline$E$ & $u(E)$ & $\ell(E)$ \\
\hline \hline integer $n$ & $|n|$ & 1 \\
\hline$E^{\prime} \pm E^{\prime \prime}$ & $u^{\prime} \ell^{\prime \prime}+\ell^{\prime} u^{\prime \prime}$ & $\ell^{\prime} \ell^{\prime \prime}$ \\
\hline$E^{\prime} \times E^{\prime \prime}$ & $u^{\prime} u^{\prime \prime}$ & $\ell^{\prime} \ell^{\prime \prime}$ \\
\hline$E^{\prime} \div E^{\prime \prime}$ & $u^{\prime} \ell^{\prime \prime}$ & $\ell^{\prime} u^{\prime \prime}$ \\
\hline$\sqrt[p]{E^{\prime}}$ & $\min \left(\sqrt[p]{u^{\prime} \ell^{\prime p-1}}, u^{\prime}\right)$ & $\min \left(\ell^{\prime}, \sqrt[p]{u^{\prime p-1} \ell^{\prime}}\right)$ \\
\hline$\diamond\left(j, F_{n}, F_{n-1}, \ldots, F_{0}\right)$ & $\Phi\left(\ldots,\left(D_{n}\right)^{i-1} D_{n-i}, \ldots\right)$ & $D_{n}$ \\
& where $D_{i}$ is given in $(7)$ & \\
\hline
\end{tabular}

The BFMSS bound constructively maintains two real parameters $u(E)$ and $\ell(E)$ as shown in Table 2. Intuitively, each expression $E$ denotes a value that can be expressed as $U(E) / L(E)$ where $U(E)$ and $L(E)$ are algebraic integers (i.e., given by division-free expressions). Then $u(E)$ (resp., $\ell(E)$ ) is an upper bound on absolute values of all the conjugates of $U(E)$ (resp., $L(E)$ ). To avoid clutter in the table, we write $u^{\prime}, u^{\prime \prime}$ for $u\left(E^{\prime}\right)$ and $u\left(E^{\prime \prime}\right)$; similarly for $\ell^{\prime}, \ell^{\prime \prime}$. Furthermore, the diamond operator involves subexpressions $F_{0}, F_{1}, \ldots, F_{n}$; in this case, we write

$$
D_{i}:=\frac{u\left(F_{i}\right)}{\ell\left(F_{i}\right)} \prod_{j=0}^{n} \ell\left(F_{j}\right) .
$$

The degree of a node in $E$ is $p$ if the node is the operator $\sqrt[p]{\cdots}$, and $n$ if the node is the diamond operator of degree $n$. Otherwise the degree is 1 . Moreover, let $D(E)$ be the product of all the degrees of the distinct nodes in the DAG of $E$. The degree of $\operatorname{val}(E)$ is bounded by $D(E)$. The BFMSS bound says that if $\operatorname{val}(E) \neq 0$ then

$$
|\operatorname{val}(E)| \geq \frac{1}{u(E)^{D(E)-1} \ell(E)} .
$$


Hence we may define the BFMSS root bound function as

$$
\beta^{\mathrm{bfmss}}(u, \ell, D):=\frac{1}{u^{D-1} \ell},
$$

with the usual convention that we write $\beta^{\mathrm{bfmss}}(E)$ for $\beta(u(E), \ell(E), D(E))$. The BFMSS Rules are given in Table 2. Our rule for $\sqrt[p]{E^{\prime}}$ in this table is a unification of the two cases in the BFMSS presentation. The advantage of having these two cases ${ }^{5}$ was shown by Yap (see [3]).

\section{Generalization of BFMSS}

Let $\alpha$ be an algebraic number. As in [8], let $\mu(\alpha)=\max \left\{\left|\alpha_{i}\right|: i=1, \ldots, n\right\}$ where $\alpha=\alpha_{1}, \ldots, \alpha_{n}$ are all conjugates of $\alpha$. We call a triple $\left(u^{\prime}, \ell^{\prime}, v\right)$ a set of $u l[2]$-parameters for $\alpha$ if $u^{\prime}, \ell^{\prime} \in \mathbb{R}_{\geq 0}$ and $v \in \mathbb{Z}$ and there exist algebraic integers $\alpha_{1}, \alpha_{2}$ such that

$$
\alpha=2^{v} \frac{\alpha_{1}}{\alpha_{2}}
$$

$\mu\left(\alpha_{1}\right) \leq u^{\prime}$ and $\mu\left(\alpha_{2}\right) \leq \ell^{\prime}$. If "2" is replaced by an integer $k>2$, we have the analogous set of $u l[k]$-parameters. When $\alpha$ is non-zero with degree $D$, we have

$$
|\alpha| \geq \beta_{2}\left(u^{\prime}, \ell^{\prime}, v, D\right):=2^{v} \frac{1}{u^{\prime D-1} \ell^{\prime}}
$$

where $\beta_{2}\left(u^{\prime}, \ell^{\prime}, v, D\right)=\beta_{2}^{\text {bfmss }}\left(u^{\prime}, \ell^{\prime}, v, D\right)$ is the binary version of the BFMSS root bound function. The expression (10) is non-unique. Indeed, there is some leeway for designing a suitable set of $u l[2]$-parameters for $\alpha$ because in general the best choice is not easily given by a fixed rule. Thus, if $\left(u^{\prime}, \ell^{\prime}, v\right)$ is a set of $u l[2]$-parameters for $\alpha$, then so is either $\left(u^{\prime} 2^{v}, \ell^{\prime}, 0\right)$ or $\left(u^{\prime}, \ell^{\prime} 2^{-v}, 0\right)$, depending on whether $v \geq 0$ or not. More generally, it is always possible to reduce $|v|$ towards 0 in any set of parameters $\left(u^{\prime}, \ell^{\prime}, v\right)$. A set of $u l[2]$-parameters is a generalization of the BFMSS parameters, since the BFMSS parameters may be regarded as the special case of $v=0$.

The BFMSS[2] Rules. The binary transformation of BFMSS is given in Table 3. The table incorporates a refinement of the $u l[2]$-parameters, whereby $v(E)$ is represented by two numbers $v^{+}(E) \geq 0$ and $v^{-}(E) \geq 0$ satisfying the

5 Namely, this modification dominates the original BFMS Rules. 
relation

$$
v(E)=v^{+}(E)-v^{-}(E) .
$$

This refinement will better quantify our gain over the original BFMSS bound (see Lemma 1 below). In actual implementation, it is sufficient to only maintain $v(E)$. In this case, to apply the rules, we will define $v^{+}(E)$ to be $v(E)$ if $v(E) \geq 0$ and otherwise let $v^{+}(E)=0$. Similarly, $v^{-}(E)$ is defined to be $-v(E)$ if $v(E)<0$ and otherwise $v^{-}(E)=0$. Call this variation the reduced version of the BFMSS[2] Rules (in contrast to the refined version where $v^{+}, v^{-}$are independent).

Table 3

The Refined BFMSS[2] Rules

\begin{tabular}{|c|c|c|c|c|}
\hline$E$ & $u_{2}=u_{2}(E)$ & $\ell_{2}=\ell_{2}(E)$ & $v^{+}=v^{+}(E)$ & $v^{-}=v^{-}(E)$ \\
\hline $\begin{array}{c}\text { binary rational } \\
n 2^{m}\end{array}$ & $|n|$ & 1 & $\max (0, m)$ & $\max (0,-m)$ \\
\hline$E^{\prime} \pm E^{\prime \prime}$ & $\begin{array}{l}2^{v^{\prime+}+v^{\prime \prime}-}-v^{+} u_{2}^{\prime} \ell_{2}^{\prime \prime} \\
+2^{v^{\prime}}+v^{\prime \prime+}{ }_{-v}^{+} \ell_{2}^{\prime} u_{2}^{\prime \prime}\end{array}$ & $\ell_{2}^{\prime} \ell_{2}^{\prime \prime}$ & $\begin{array}{r}\min \left(v^{\prime+}+v^{\prime \prime-},\right. \\
\left.v^{\prime-}+v^{\prime \prime+}\right) \\
\end{array}$ & $v^{\prime-}+v^{\prime \prime-}$ \\
\hline$E^{\prime} \times E^{\prime \prime}$ & $u_{2}^{\prime} u_{2}^{\prime \prime}$ & $\ell_{2}^{\prime} \ell_{2}^{\prime \prime}$ & $v^{\prime+}+v^{\prime \prime+}$ & $v^{\prime-}+v^{\prime \prime-}$ \\
\hline$E^{\prime} \div E^{\prime \prime}$ & $u_{2}^{\prime} \ell_{2}^{\prime \prime}$ & $\ell_{2}^{\prime} u_{2}^{\prime \prime}$ & $v^{\prime+}+v^{\prime \prime-}$ & $v^{\prime-}+v^{\prime \prime+}$ \\
\hline$\sqrt[p]{E^{\prime}}, 2^{v^{\prime}} u_{2}^{\prime} \geq \ell_{2}^{\prime}$ & $\sqrt[p]{2^{\tilde{v}-p v^{+}} u_{2}^{\prime} \ell_{2}^{\prime p-1}}$ & $\ell_{2}^{\prime}$ & $\begin{array}{c}\tilde{v} / p] \text { where } \\
\widetilde{v}=v^{\prime+}+(p-1) v^{\prime-}\end{array}$ & $v^{\prime-}$ \\
\hline$\sqrt[p]{E^{\prime}}, 2^{v^{\prime}} u_{2}^{\prime}<\ell_{2}^{\prime}$ & $u_{2}^{\prime}$ & $\sqrt[p]{2^{\tilde{v}-p v^{-} u_{2}^{\prime p-1} \ell_{2}^{\prime}}}$ & $v^{\prime+}$ & $\begin{array}{l}\widetilde{v}=\left[\begin{array}{l}{[v / p} \\
\tilde{v}\end{array}=(p-1) v^{\prime+}+v^{\prime-}\right.\end{array}$ \\
\hline$\diamond\left(j ; F_{n},, \ldots, F_{0}\right)$ & $\begin{array}{c}\Phi\left(\ldots, C_{n}^{i-1} C_{n-i}, \ldots\right) \\
(\text { see }(14))\end{array}$ & $2^{-w_{n}} C_{n}$ & 0 & $w_{n} \quad($ see $\quad(13))$ \\
\hline
\end{tabular}

When $\alpha$ is represented by an expression $E$ (in the DAG form), this table defines a unique set of $u l[2]$-parameters for $E$,

$$
\left(u_{2}(E), \ell_{2}(E), v(E)\right) .
$$

The BFMSS[2] root bound for $E$ is

$$
E \neq 0 \Rightarrow|E| \geq \frac{2^{v(E)}}{u_{2}(E)^{D(E)-1} \ell_{2}(E)}
$$

In the table, $\left(u^{\prime}, \ell^{\prime}, v^{\prime}\right)$ denotes the $u l[2]$-parameters of the subexpression $E^{\prime}$; similarly $\left(u^{\prime \prime}, \ell^{\prime \prime}, v^{\prime \prime}\right)$ is for $E^{\prime \prime}$.

Most of the rules in Table 3 can be read off the table; but the more complex diamond operator will be explained here. We want a set of $u l[2]$-parameters for $\diamond\left(j ; F_{n}, F_{n-1}, \ldots, F_{0}\right)$. Suppose $\Phi\left(a_{n-1}, a_{n-2}, \ldots, a_{0}\right)$ is a root bound function, as in (6). Write $v_{i}$ for $v_{i}^{+}-v_{i}^{-}=v^{+}\left(F_{i}\right)-v_{i}^{-}\left(F_{i}\right)$. Define

$$
\begin{aligned}
w_{i} & :=v_{i}+\left(\sum_{j=0}^{n} v_{j}^{-}\right) \\
& =v_{0}^{-}+\cdots+v_{i-1}^{-}+v_{i}^{+}+v_{i+1}^{-}+\cdots+v_{n}^{-}
\end{aligned}
$$


and

$$
C_{i}=2^{w_{i}} \frac{u_{2}\left(F_{i}\right)}{\ell_{2}\left(F_{i}\right)} \prod_{j=0}^{n} \ell_{2}\left(F_{j}\right)
$$

Just as in BFMSS, the diamond operator (if well-defined) $\diamond\left(j ; F_{n}, F_{n-1}, \ldots, F_{0}\right)$ specifies an algebraic number $\alpha$ where $\alpha=U / L$ and $U, L$ are algebraic integers satisfying

$$
\mu(U) \leq \Phi\left(\ldots,\left(C_{n}\right)^{i-1} C_{n-i}, \ldots\right), \quad \mu(L) \leq C_{n}
$$

Also, a set of $u l[2]$-parameters for $\alpha$ is

$$
\left(\Phi\left(\ldots,\left(C_{n}\right)^{i-1} C_{n-i}, \ldots\right), 2^{-w_{n}} C_{n},-w_{n}\right) .
$$

This justifies the rule for diamond operator in Table 3 (other rules will be justified below).

If we know more about the nature of $\Phi$, improved bounds may be possible. E.g., using the Lagrange-Zassenhaus bound [15], we get the simpler set of $u l[2]$-parameters,

$$
\left(\Phi\left(\ldots, D_{n-i}, \ldots\right), 1,0\right)
$$

where $D_{n-i}$ is given by $(7)$.

BFMSS[2] dominates BFMSS. We first prove a key relationship between the BFMSS Rules and the new BFMSS[2] Rules.

LEMMA 1 Let

$$
(u, \ell), \quad\left(u_{2}, \ell_{2}, v^{+}, v^{-}\right)
$$

be the parameters for an expression $E$ given by Table 2 and Table 3, respectively. Then

$$
u=2^{v^{+}} u_{2}, \quad \ell=2^{v^{-}} \ell_{2} .
$$

PROOF. We use induction on the structure of $E$. The base case is obvious. 
CASE $E=E^{\prime} \pm E^{\prime \prime}$ :

$$
\begin{aligned}
\frac{u}{\ell} & =\frac{u^{\prime} \ell^{\prime \prime}+\ell^{\prime} u^{\prime \prime}}{\ell^{\prime} \ell^{\prime \prime}} \\
& =\frac{2^{v^{+}+v^{\prime \prime}-} u_{2}^{\prime} \ell_{2}^{\prime \prime}+2^{v^{\prime-}+v^{\prime \prime+}} \ell_{2}^{\prime} u_{2}^{\prime \prime}}{2^{v^{\prime-}+v^{\prime \prime}-} \ell_{2}^{\prime} \ell_{2}^{\prime \prime}} \\
& =\frac{2^{v^{+}}\left(2^{v^{\prime+}+v^{\prime \prime}-v^{+}} u_{2}^{\prime} \ell_{2}^{\prime \prime}+2^{v^{\prime}+}+v^{\prime \prime+}-v^{+} \ell_{2}^{\prime} u_{2}^{\prime \prime}\right)}{2^{v^{\prime}+}+v^{\prime \prime}-\ell_{2}^{\prime} \ell_{2}^{\prime \prime}} \\
& =\frac{2^{v^{+}} u_{2}}{2^{v-} \ell_{2}}
\end{aligned}
$$

where $v^{+}=\min \left(v^{\prime+}+v^{\prime \prime-}, v^{\prime-}+v^{\prime \prime-}\right)$ and $v^{-}=v^{-}+v^{\prime \prime-}$. We want to conclude from this derivation that

$$
u=2^{v^{+}} u_{2}, \quad \ell=2^{v^{-}} \ell_{2} .
$$

This is only valid if, in the above derivation, we never apply any cancellation rules between the numerator and denominator. The reader may verify this is the case. In other words, although we presented the argument as a sequence of equations involving ratios, it should be read as a pair of parallel transformations involving the numerator and denominator separately. This will also be true in all the other derivations in this proof.

CASE $E=E^{\prime} \times E^{\prime \prime}$ :

$$
\begin{aligned}
& \frac{u}{\ell}=\frac{u^{\prime} u^{\prime \prime}}{\ell^{\prime} \ell^{\prime \prime}} \\
& =\frac{2^{v^{++}+v^{\prime \prime}+} u_{2}^{\prime} u_{2}^{\prime \prime}}{2^{v^{\prime-}+v^{\prime \prime}-} \ell_{2}^{\prime} \ell_{2}^{\prime \prime}} \text { (induction) } \\
& =\frac{2^{v^{+}} u_{2}}{2^{v^{-}} \ell_{2}} \quad \text { (BFMSS[2]) }
\end{aligned}
$$

where $v^{+}=v^{\prime+}+v^{\prime \prime+}$ and $v^{-}=v^{\prime-}+v^{\prime \prime-}$. The division case is similar.

CASE $E=E^{\prime} \div E^{\prime \prime}$ :

$$
\begin{aligned}
& \frac{u}{\ell}=\frac{u^{\prime} \ell^{\prime \prime}}{\ell^{\prime} u^{\prime \prime}} \\
& =\frac{2^{v^{\prime+}+v^{\prime \prime}-} u_{2}^{\prime} \ell_{2}^{\prime \prime}}{2^{v^{\prime}+v^{\prime \prime}} \ell_{2}^{\prime} u_{2}^{\prime \prime}} \text { (induction) } \\
& =\frac{2^{v^{+}} u_{2}}{2^{v^{-}} \ell_{2}} \quad \text { (BFMSS[2]) }
\end{aligned}
$$

where $v^{+}=v^{\prime+}+v^{\prime \prime-}$ and $v^{-}=v^{\prime-}+v^{\prime \prime}$.

CASE $E=\sqrt[p]{E^{\prime}}$ : The rules here split into two cases, depending on whether or not $2^{v} u_{2}^{\prime} \geq \ell_{2}^{\prime}$. The critical observation is that $2^{v} u_{2}^{\prime} \geq \ell_{2}^{\prime}$ is equivalent to $u^{\prime} \geq \ell^{\prime}$ (the corresponding criterion for choosing between the two cases in the BFMSS Rule). First assume $2^{v^{\prime}} u_{2}^{\prime} \geq \ell_{2}^{\prime}$. Let $\widetilde{v}=v^{\prime+}+(p-1) v^{\prime-}, v^{+}=\lfloor\widetilde{v} / p\rfloor$ 
and $v^{-}=v^{-}$. We have

$$
\begin{array}{rlr}
\frac{u}{\ell} & =\frac{\sqrt[p]{u^{\prime} \ell^{\prime p-1}}}{\ell^{\prime}} & (\text { BFMSS }) \\
& =\frac{\sqrt[p]{2^{v^{+}+(p-1) v^{\prime-}} u_{2}^{\prime} \ell_{2}^{\prime p-1}}}{2^{v^{\prime}} \ell_{2}^{\prime}} & (\text { induction }) \\
& =\frac{2^{v^{+}} u_{2}}{2^{v^{-}} \ell_{2}} & (\text { BFMSS }[2]) .
\end{array}
$$

The other case, when $2^{v^{\prime}} u_{2}^{\prime}<\ell_{2}^{\prime}$ is similar but not shown.

CASE $E=\diamond\left(F_{n}, \ldots, F_{0}\right)$ : For $i=0, \ldots, n$, we have

$$
\begin{aligned}
& D_{i}=\frac{u\left(F_{i}\right)}{\ell\left(F_{i}\right)} \prod_{j=0}^{n} \ell\left(F_{j}\right) \quad(\mathrm{BFMSS}) \\
& =2^{w_{i}} \frac{u_{2}\left(F_{i}\right)}{\ell_{2}\left(F_{i}\right)} \prod_{j=0}^{n} \ell_{2}\left(F_{j}\right) \text { (induction) } \\
& =C_{i} \quad(\operatorname{BFMSS}[2]) \text {. }
\end{aligned}
$$

Thus

$$
\begin{aligned}
u(E) & =\Phi\left(\ldots,\left(D_{n}\right)^{i-1} D_{n-i}, \ldots\right) \\
& =\Phi\left(\ldots,\left(C_{n}\right)^{i-1} C_{n-i}, \ldots\right) \\
& =u_{2}(E)=2^{v^{+}} u_{2}(E) .
\end{aligned}
$$

Similarly, $\ell(E)=D_{n}=C_{n}=2^{w_{n}} \ell_{2}(E)=2^{v^{-}} \ell_{2}(E)$.

Our main result concerning the BFMSS and BFMSS[2] Rules is the following domination relation:

TheOREM 2 For any expression E supported by Table 2, we have

$$
\beta_{2}^{\text {bfmss }}(E) \geq \beta^{\text {bfmss }}(E) .
$$

PROOF. Let $\beta(E)=\frac{1}{u^{D-1} \ell}$ and $\beta_{2}=\frac{2^{v}}{u_{2}^{D-1} \ell_{2}}$ be (respectively) the BFMSS and BFMSS[2] bounds for expression E. From Lemma 1, we conclude

$$
\frac{\beta_{2}}{\beta}=\frac{2^{v} \cdot\left(2^{v^{+}} u_{2}\right)^{D-1} \cdot\left(2^{v^{-}} \ell_{2}\right)}{u_{2}^{D-1} \ell_{2}}=2^{v^{+} D} \geq 1
$$

Correctness and the Umbral Convention. We now justify the BFMSS[2] Rules in Table 3 . The correctness of a set $\left(u_{2}, \ell_{2}, v\right)$ of $u l[2]$-parameters for an expression $E$ depends on the existence of algebraic integers $U_{2}, L_{2}$ such that

$$
E=2^{v} \frac{U_{2}}{L_{2}}
$$


with $u_{2} \geq \mu\left(U_{2}\right), \ell_{2} \geq \mu\left(L_{2}\right)$. We have not given explicit rules for maintaining $U_{2}, L_{2}$, but these are easily deduced from Table 3 . That is because the rules for maintaining $u_{2}, \ell_{2}$ is a "shadow" of the corresponding rules for $U_{2}, L_{2}$. Let us illustrate this: when $E=E^{\prime} \pm E^{\prime \prime}$, we have the rule

$$
u_{2}=2^{v^{\prime+}+v^{\prime \prime-}-v^{+}} u_{2}^{\prime} \ell_{2}^{\prime \prime}+2^{v^{\prime-}+v^{\prime \prime+}-v^{+}} \ell_{2}^{\prime} u_{2}^{\prime \prime}
$$

This is a "shadow" of the corresponding ${ }^{6}$ rule for $U_{2}$ :

$$
U_{2}=2^{v^{\prime+}+v^{\prime \prime-}-v^{+}} U_{2}^{\prime} L_{2}^{\prime \prime} \pm 2^{v^{\prime-}+v^{\prime \prime+}-v^{+}} L_{2}^{\prime} U_{2}^{\prime \prime} .
$$

REMARKS: The original BFMSS rules also have such an umbral connection between $(u, \ell)$ and the pair of expressions $(U, L)$, although this was only implicit. Such a shadowing technique is similar to the mnemonic device called symbolic or "umbral calculus" from the invariant theorists, and developed by Rota and his collaborators [11] as a form of linear operator.

The umbral relation between $\left(u_{2}, \ell_{2}\right)$ and $\left(U_{2}, L_{2}\right)$ is justified by the following:

LEMma 3 For any expression $E$,

(i) The expressions $U_{2}(E)$ and $L_{2}(E)$ are algebraic integers.

(ii) The following inequalities hold:

$$
u_{2} \geq \mu\left(U_{2}\right), \quad \ell_{2} \geq \mu\left(L_{2}\right) .
$$

PROOF. (i) We sketch the justification of the rules for $U_{2}(E)$; the justification of $L_{2}(E)$ is analogous. Consider the case when $E=E^{\prime} \pm E^{\prime \prime}$. Then $U_{2}(E)$ is given by (18), and this is an algebraic integer because $v^{\prime+}+v^{\prime \prime-}-v^{+} \geq 0$ and $v^{\prime-}+v^{\prime \prime+}-v^{+} \geq 0$ (also, inductively, the subexpressions $U_{2}^{\prime}, U_{2}^{\prime \prime}$ are algebraic integers). In the case of radicals, we use the fact that $\sqrt[p]{E^{\prime}}$ is an algebraic integer when $E^{\prime}$ is an algebraic integer. The remaining cases are just as easily shown. (ii) We sketch the argument for part (ii). The relationship (19) holds because for algebraic integers $A, B$, if $a \geq \mu(A)$ and $b \geq \mu(B)$ then

$$
a+b \geq \mu(A \pm B), \quad a b \geq \mu(A B), \quad \sqrt[p]{a} \geq \mu(\sqrt[p]{A})
$$

In particular, this justifies why (17) is an upper bound on the algebraic integer (18).

We are ready to prove the correctness of our rules.

\footnotetext{
${ }_{6}$ Note that the rules for $u_{2}, \ell_{2}$ shadow the rules for $U_{2}, L_{2}$, but not vice-versa, because \pm for $U_{2}, L_{2}$ becomes a + for $u_{2}, \ell_{2}$. This can be seen by comparing (17) and (18).
} 
THEOREM 4 Table 3 is correct: for each expression E, the triple $\left(u_{2}(E), \ell_{2}(E), v(E)\right)$ is a set of $u l[2]$-parameters for $E$.

PROOF. Since we already know Lemma 3, it remains to show the relation (16). The BFMSS rules produce a pair of algebraic integer expressions $U(E), L(E)$ such that $E=U(E) / L(E)$. Lemma 1 shows that

$$
\frac{u}{\ell}=\frac{2^{v^{+}} u_{2}}{2^{v^{-}} \ell_{2}}
$$

From the umbral relation between $(u, \ell)$ and $(U, L)$, and also between $\left(u_{2}, \ell_{2}\right)$ and $\left(U_{2}, L_{2}\right)$, we conclude that

$$
\frac{U}{L}=\frac{2^{v^{+}} U_{2}}{2^{v^{-}} L_{2}}=2^{v} \frac{U_{2}}{L_{2}}
$$

Generalization. We can generalize the $u l[2]$-parameters to $u l[k]$-parameters for any integer $k>2$. Since the majority of input constants in scientific and engineering computations is covered by the $u l[2]$ or $u l[10]$, the following generalization will be useful: if $q_{1}, \ldots, q_{n} \geq 2$ are relatively prime, it is easy to define a set

$$
\left(u(E), \ell(E), v_{q_{1}}(E), \ldots, v_{q_{n}}(E)\right)
$$

of $u l\left[q_{1}, \ldots, q_{n}\right]$-parameters for $E$, so that

$$
E=\frac{u(E)}{l(E)} \prod_{i=1}^{n} q_{i}^{v_{q_{i}}}
$$

Special Cases. The binary BFMSS Rules allow the root bounds of a floating point constant to behave like an integer (i.e., $\ell(E)=1$ ). As long as there is no explicit division in our expression, the expression continues to behave like an integer. This is a very important case in practice.

Let us consider some specialization of our rules. Suppose $E^{\prime}$ and $E^{\prime \prime}$ are "almost division-free" in the sense that $\ell_{2}^{\prime}=\ell_{2}^{\prime \prime}=1$ (they may not be algebraic integers since $v^{\prime}, v^{\prime \prime}$ can be negative). Then the rule for $E=E^{\prime} \pm E^{\prime \prime}$ in Table 3 gives

$$
u_{2}=2^{v^{\prime+}+v^{\prime \prime-}-v^{+}} u_{2}^{\prime}+2^{v^{\prime-}+v^{\prime \prime+}-v^{+}} u_{2}^{\prime \prime} .
$$

When $v^{\prime}=v^{\prime \prime}$, this further simplifies to $u_{2}=u_{2}^{\prime}+u_{2}^{\prime \prime}$. Similarly $\ell_{2}=1$ and $v=v^{\prime}$. Suppose $x, y$ are two $L$-bit binary numbers. Such numbers can be represented by a binary string of length $L$ with a binary point somewhere in the string. So the triple $\left(4^{L}, 1,-L\right)$ is a set of $u l[2]$-parameters for $x$ and for $y$. From the preceding, $x+y$ has $u l[2]$-parameters $\left(2 \cdot 4^{L}, 1,-L\right)$. Similarly, $x y$ 
has the $u l[2]$-parameters $\left(4^{2 L}, 1,-2 L\right)$. Now suppose $E$ is the determinant of an $n \times n$ matrix with entries which are $L$-bit binary numbers. Viewing $E$ as the standard sum of $n$ ! terms, we easily see that $E$ has

$$
\left(4^{n L} n !, 1,-n L\right)
$$

as a set of $u l[2]$-parameters. Furthermore, since $D(E)=1, \beta_{2}^{\text {bfmss }}(E)=2^{-n L}$. This justifies the root bit bound given in (2).

\section{The $k$-ary Measure Bound}

The Measure Bound Rules from Li-Yap [8] (cf. [10,1]) is shown in the first two columns of Table 4. For each expression $E$, it maintains $\mathrm{M}(E)$ according to the table. The degree bound $D(E)$ is independently computed as usual. If $E^{\prime}$ is a subexpression, we write $\mathrm{M}^{\prime}$ and $D^{\prime}$ for $\mathrm{M}\left(E^{\prime}\right)$ and $D\left(E^{\prime}\right)$; similarly for $\mathrm{M}^{\prime \prime}$ and $D^{\prime \prime}$.

Notice that Line 7 refers $^{7}$ to the $\operatorname{Root}\left(F_{n}, \ldots, F_{0}\right)$ operator. This is just a special case of the diamond operator of BFMSS in which the $F_{i}$ 's are now explicit integers. Thus, such root operators are (essentially) leafs in an expression DAG. This case is not only the most important, it is the version for which efficient algorithms exist. The general diamond operator does not seem easy to implement. Again, we use any inclusion bounding function $\Phi$. Extracting $v(E)$ from the arguments $F_{i}$ is relatively straightforward in practice, assuming a binary representation of integers.

The binary version of the measure bound is shown in the last two columns of Table 4. For each expression $E$, this maintains $v(E)$ and $\mathrm{M}_{2}(E)$. Note that in Line $5\left(E=\sqrt[p]{E^{\prime}}\right)$, we choose $v$ to be the rounding of $v^{\prime} / p$ towards 0 , to simplify the proof of Lemma 6.

Binary Measure Parameters. Call $(x, y) \in \mathbb{Z} \times \mathbb{R}_{\geq 1}$ a set of binary measure parameters for an expression $E$ if there exists an expression $E_{2}$ such that

$$
E=2^{x} E_{2}, \quad \mathrm{M}\left(E_{2}\right) \leq y .
$$

Lemma 5 If $v(E)$ and $\mathrm{M}_{2}(E)$ are defined as in Table 4, then $\left(v(E), \mathrm{M}_{2}(E)\right)$ is a set of binary measure parameters for $E$.

\footnotetext{
7 Like the diamond operator, one must also specify some parameter to identify a particular root of the polynomial. This extra parameter is omitted here since the root bounds do not depend on it.
} 
Table 4

Binary Measure Bound

\begin{tabular}{|c|c|c|c|c|}
\hline & $E$ & $\mathrm{M}(E)$ & $v(E)$ & $\mathrm{M}_{2}(E)$ \\
\hline 0 & Constant 1 & 1 & 0 & 1 \\
\hline 1 & $2^{v^{\prime}} \frac{a}{b} E^{\prime \prime}\left(v^{\prime} \in \mathbb{Z}\right)$ & $\begin{array}{l}\max \left\{2^{v^{\prime+}}|a|\right. \\
\left.2^{v^{\prime-}}|b|\right\}^{D^{\prime \prime}} \mathrm{M}^{\prime \prime}\end{array}$ & $v^{\prime}+v^{\prime \prime}$ & $\max \{|a|,|b|\}^{D^{\prime \prime}} \mathrm{M}_{2}^{\prime \prime}$ \\
\hline 2 & $E^{\prime} \times E^{\prime \prime}$ & $\mathrm{M}^{\prime D^{\prime \prime}} \mathrm{M}^{\prime \prime D^{\prime}}$ & $v^{\prime}+v^{\prime \prime}$ & $\mathrm{M}_{2}^{\prime D^{\prime \prime}} \mathrm{M}_{2}^{\prime \prime D^{\prime}}$ \\
\hline 3 & $E^{\prime} \div E^{\prime \prime}$ & $\mathrm{M}^{\prime D^{\prime \prime}} \mathrm{M}^{\prime \prime D^{\prime}}$ & $v^{\prime}-v^{\prime \prime}$ & $\mathrm{M}_{2}^{\prime D^{\prime \prime}} \mathrm{M}_{2}^{\prime \prime D^{\prime}}$ \\
\hline 4 & $E^{\prime} \pm E^{\prime \prime}$ & $\mathrm{M}^{\prime D^{\prime \prime}} \mathrm{M}^{\prime \prime D^{\prime}} 2^{D(E)}$ & $\begin{array}{l}v=\operatorname{sign}\left(v^{\prime}\right) \min \left\{\left|v^{\prime}\right|,\left|v^{\prime \prime}\right|\right\} \\
\text { if } v^{\prime} v^{\prime \prime} \geq 0 ; \text { else } v=0\end{array}$ & $\begin{array}{l}2^{D+d} \mathrm{M}_{2}^{\prime D^{\prime \prime}} \mathrm{M}_{2}^{\prime \prime D^{\prime}} \\
d=\left|v^{\prime}-v^{\prime \prime}\right| D^{\prime} D^{\prime \prime}\end{array}$ \\
\hline 5 & Radical $\sqrt[p]{E^{\prime}}$ & $\mathrm{M}^{\prime}$ & $\begin{array}{l}v=\left\lfloor v^{\prime} / p\right\rfloor \text { if } v^{\prime} \geq 0 \\
v=\left\lceil v^{\prime} / p\right\rceil \text { else. }\end{array}$ & $\begin{array}{l}2^{|m| D^{\prime}} \mathrm{M}_{2}^{\prime} \\
m=v^{\prime}-v p\end{array}$ \\
\hline 6 & Power $E^{\prime k}$ & $\mathrm{M}^{\prime k}$ & $v^{\prime} k$ & $\mathrm{M}_{2}^{\prime k}$ \\
\hline 7 & $\begin{array}{l}\operatorname{Root}\left(F_{n}, \ldots, F_{0}\right) \\
F_{i}=2^{v_{i}} a_{i} \\
v_{i} \geq 0(0 \leq i \leq n)\end{array}$ & $\Phi\left(\frac{F_{n-1}}{F_{n}}, \ldots, \frac{F_{0}}{F_{n}}\right)$ & $v=-\max \left\{d: v_{i} \geq i d, 1 \leq i \leq n\right\}$ & $\begin{array}{l}\Phi\left(\frac{f_{n-1}}{f_{n}}, \ldots, \frac{f_{0}}{f_{n}}\right) \text {, } \\
\text { and } f_{i}=2^{v_{i}+i v} a_{i} .\end{array}$ \\
\hline
\end{tabular}

Table 5

Correctness of Binary Measure Bound

\begin{tabular}{|c|c|c|c|c|}
\hline & $E$ & $v(E)$ & $\mathrm{M}_{2}(E)$ & $E_{2}$ \\
\hline 0 & Constant 1 & 0 & 1 & 1 \\
\hline 1 & $2^{v^{\prime}} \frac{a}{b} E^{\prime \prime}\left(v^{\prime} \in \mathbb{Z}\right)$ & $v^{\prime}+v^{\prime \prime}$ & $\max \{|a|,|b|\}^{D^{\prime \prime}} \mathrm{M}_{2}^{\prime \prime}$ & $\frac{a}{b} E_{2}^{\prime \prime}$ \\
\hline 2 & $E^{\prime} \times E^{\prime \prime}$ & $v^{\prime}+v^{\prime \prime}$ & $\mathrm{M}_{2}^{\prime D^{\prime \prime}} \mathrm{M}_{2}^{\prime \prime D^{\prime}}$ & $E_{2}^{\prime} \times E_{2}^{\prime \prime}$ \\
\hline 3 & $E^{\prime} \div E^{\prime \prime}$ & $v^{\prime}-v^{\prime \prime}$ & $\mathrm{M}_{2}^{\prime D^{\prime \prime}} \mathrm{M}_{2}^{\prime \prime D^{\prime}}$ & $E_{2}^{\prime} \div E_{2}^{\prime \prime}$ \\
\hline 4 & $E^{\prime} \pm E^{\prime \prime}$ & $\begin{array}{l}v=\operatorname{sign}\left(v^{\prime}\right) \min \left\{\left|v^{\prime}\right|,\left|v^{\prime \prime}\right|\right\} \\
\text { if } v^{\prime} v^{\prime \prime} \geq 0 ; \text { else } v=0\end{array}$ & $\begin{array}{c}2^{D(E)+d} \mathrm{M}_{2}^{\prime D^{\prime \prime}} \mathrm{M}_{2}^{\prime \prime D^{\prime}} \\
d=\left|v^{\prime}-v^{\prime \prime}\right| D^{\prime} D^{\prime \prime}\end{array}$ & $2^{v^{\prime}-v} E_{2}^{\prime}+2^{v^{\prime \prime}-v} E_{2}^{\prime \prime}$ \\
\hline 5 & Radical $\sqrt[p]{E^{\prime}}$ & $\begin{array}{l}v=\left\lfloor v^{\prime} / p\right\rfloor \text { if } v^{\prime} \geq 0 \\
v=\left\lceil v^{\prime} / p\right\rceil \text { else. }\end{array}$ & $\begin{array}{c}2^{|m| D^{\prime}} \mathrm{M}_{2}^{\prime} \\
m=v^{\prime}-v p\end{array}$ & $\sqrt[p]{2^{m} E_{2}^{\prime}}$ \\
\hline 6 & Power $E^{\prime k}$ & $v^{\prime} k$ & $\mathrm{M}_{2}^{\prime k}$ & $E_{2}^{\prime k}$ \\
\hline 7 & $\begin{array}{l}\operatorname{Root}\left(F_{n}, \ldots, F_{0}\right) \\
F_{i}=2^{v_{i}} a_{i} \\
v_{i} \geq 0(0 \leq i \leq n)\end{array}$ & $v=-\max \left\{d: v_{i} \geq i d, 1 \leq i \leq n\right\}$ & $\begin{array}{l}\Phi\left(\frac{f_{n-1}}{f_{n}}, \ldots, \frac{f_{0}}{f_{n}}\right) \\
\text { and } f_{i}=2^{v_{i}+i v} a_{i}\end{array}$ & $\operatorname{Root}\left(f_{n}, \ldots, f_{0}\right)$ \\
\hline
\end{tabular}

PROOF. We first claim that $E=2^{v(E)} E_{2}$ for a suitable $E_{2}$. We augment Table 4 with another column showing how $E_{2}$ is defined inductively; the result is displayed as Table 5 .

Most of the entries in the last column are straightforward, so we skip the verification. We only verify two cases for $\mathrm{M}_{2}(E)$ :

Line $4, E=E^{\prime} \pm E^{\prime \prime}$. Here, we have $E_{2}=2^{v^{\prime}-v} E_{2}^{\prime}+2^{v^{\prime \prime}-v} E_{2}^{\prime \prime}$, and based on the Measure Rules, we can compute $\mathrm{M}\left(2^{v^{\prime}-v} E_{2}^{\prime}\right)=2^{\left|v^{\prime}-v\right| D^{\prime}} \mathrm{M}_{2}^{\prime}$ and $\mathrm{M}\left(2^{v^{\prime \prime}-v} E_{2}^{\prime \prime}\right)=$ 
$2^{\left|v^{\prime \prime}-v\right| D^{\prime \prime}} \mathrm{M}_{2}^{\prime \prime}$. Hence

$$
\begin{aligned}
\mathrm{M}\left(2^{v^{\prime}-v} E_{2}^{\prime}+2^{v^{\prime \prime}-v} E_{2}^{\prime \prime}\right) & =2^{D} \mathrm{M}\left(2^{v^{\prime}-v} E_{2}^{\prime}\right)^{D^{\prime \prime}} \mathrm{M}\left(2^{v^{\prime \prime}-v} E_{2}^{\prime \prime}\right)^{D^{\prime}} \\
& =2^{D+\left(\left|v^{\prime}-v\right|+\left|v^{\prime \prime}-v\right|\right) D^{\prime} D^{\prime \prime}} \mathrm{M}\left(E_{2}^{\prime}\right)^{D^{\prime \prime}} \mathrm{M}\left(E_{2}^{\prime \prime}\right)^{D^{\prime}} \\
& =2^{D+\left|v^{\prime}-v^{\prime \prime}\right| D^{\prime} D^{\prime \prime}} \mathrm{M}\left(E_{2}^{\prime}\right)^{D^{\prime \prime}} \mathrm{M}\left(E_{2}^{\prime \prime}\right)^{D^{\prime}} \\
& =2^{D+d} \mathrm{M}\left(E_{2}^{\prime}\right)^{D^{\prime \prime}} \mathrm{M}\left(E_{2}^{\prime \prime}\right)^{D^{\prime}}
\end{aligned}
$$

This justifies the rule for $M_{2}\left(E^{\prime} \pm E^{\prime \prime}\right)$.

Line $7, E=\operatorname{Root}\left(F_{n}, \ldots, F_{0}\right)$ where each $F_{i}=2^{v_{i}} a_{i}$ and $v_{i} \geq 0$. Let $P(X)=\sum_{i=0}^{n} F_{i} X^{i}$. From $E=2^{v} E_{2}$, we conclude that $E_{2}$ is a root of the polynomial $Q(X)=P\left(2^{v} X\right)=\sum_{i=0}^{n} 2^{v_{i}+i v} a_{i} X^{i}$. But $v=-\max \left\{d: v_{i} \geq i d, i=\right.$ $1, \ldots, n\} \geq 0$ implies that $Q(X)=\sum_{i=0}^{n} 2^{v_{i}+i v} a_{i} X^{i}$ is an integer polynomial. With $f_{i}=2^{v_{i}+i v} a_{i}$, we see that $\mathrm{M}_{2}(E)=\mathrm{M}\left(E_{2}\right)=\Phi\left(f_{n-1} / f_{n}, \ldots, f_{0} / f_{n}\right)$ is a valid rule. 
Domination. Let $\beta(E)$ and $\beta_{2}(E)$ be the root bound functions associated with the original measure bound and the binary measure bound. Indeed,

$$
\beta(E)=\frac{1}{\mathrm{M}(E)}, \quad \beta_{2}(E)=\frac{2^{v(E)}}{\mathrm{M}_{2}(E)} .
$$

It is easy to see that $\beta_{2}(E)$ is a root bound for $E$ since $E \neq 0$ implies

$$
\begin{aligned}
|E| & =2^{v(E)}\left|E_{2}\right| \quad\left(\text { by validity of } \beta_{2}\right) \\
& \geq 2^{v(E)} / \mathrm{M}\left(E_{2}\right) \quad(\text { the usual measure bound) } \\
& \geq 2^{v(E)} / \mathrm{M}_{2}(E) \quad\left(\text { since } \mathrm{M}\left(E_{2}\right) \leq \mathrm{M}_{2}(E), \text { putting } y=M_{2}(E) \text { in }(22)\right) \\
& =\beta_{2}(E) .
\end{aligned}
$$

Our goal is to prove that $\beta_{2}$ dominates $\beta$ in the sense that $\beta_{2}(E) \geq \beta(E)$ for all $E$ supported by the operators in Table 4 . Unfortunately, we cannot do this without further information about the root bound function $\Phi(\cdots)$. We get around this problem by modifying Line 7 in Table 4 so that $v(E)=0$ and $M_{2}(E)=M(E)$. Call this the "trivial rule" for the root operator. We prove a basic inequality:

Lemma 6 Let $E$ be any expression involving the operators of Table 4. Assuming the trivial rule for the root operator, we have

$$
\mathrm{M}(E) \geq 2^{|v(E)| D(E)} \mathrm{M}_{2}(E) .
$$

PROOF. We verify this lemma for each line of the Table 4 .

Line 0 . When $E=1$, the lemma is immediate since $v(E)=0$ and $\mathrm{M}(E)=$ $\mathrm{M}_{2}(E)$.

Line 1. Actually, Line 1 is a special case of Line 2, with $E^{\prime}=2^{v^{\prime}} a / b$ (so $\left.D^{\prime}=1\right)$. So we just have to check that $M_{2}^{\prime}=\mathrm{M}(a / b)=\max \{|a|,|b|\}$.

Line $2, E=E^{\prime} E^{\prime \prime}$.

$$
\begin{aligned}
& \mathrm{M}(E)=\mathrm{M}^{\prime D^{\prime \prime}} \mathrm{M}^{\prime \prime D^{\prime}} \quad \text { (Measure Rules) } \\
& \geq 2^{\left(\left|v^{\prime}\right|+\left|v^{\prime \prime}\right|\right) D^{\prime} D^{\prime \prime}} \mathrm{M}_{2}^{\prime D^{\prime \prime}} \mathrm{M}_{2}^{\prime \prime D^{\prime}} \text { (by induction) } \\
& \geq 2^{\left|v^{\prime}+v^{\prime \prime}\right| D} \mathrm{M}_{2}^{\prime D^{\prime \prime}} \mathrm{M}_{2}^{\prime \prime D^{\prime}} \quad\left(\left|v^{\prime}\right|+\left|v^{\prime \prime}\right| \geq\left|v^{\prime}+v^{\prime \prime}\right|, D^{\prime} D^{\prime \prime} \geq D\right) \\
& =2^{|v| D} \mathrm{M}_{2}(E) \quad \text { (Measure[2] Rules). }
\end{aligned}
$$

Line 3, E $=E^{\prime} / E^{\prime \prime}$. This is similar to the proof of Line 2, since the usual Measure Rules for division is exactly the same as for multiplication. However, in the Measure[2] Rules, $v=v^{\prime}-v^{\prime \prime}$. This changes one justification in the preceding proof, replacing $\left|v^{\prime}\right|+\left|v^{\prime \prime}\right| \geq\left|v^{\prime}+v^{\prime \prime}\right|=|v|$ by $\left|v^{\prime}\right|+\left|v^{\prime \prime}\right| \geq\left|v^{\prime}-v^{\prime \prime}\right|=$ 
$|v|$.

Line $4, E=E^{\prime} \pm E^{\prime \prime}$.

$$
\begin{array}{rlrl}
\mathrm{M}(E) & =2^{D} \mathrm{M}^{\prime D^{\prime \prime}} \mathrm{M}^{\prime \prime D^{\prime}} & & \text { (Measure Rules) } \\
& \geq 2^{D+\left(\left|v^{\prime}\right|+\left|v^{\prime \prime}\right|\right) D^{\prime} D^{\prime \prime}} \mathrm{M}_{2}^{\prime D^{\prime \prime}} \mathrm{M}_{2}^{\prime \prime D^{\prime}} & \text { (by induction) } \\
& \geq 2^{D+\left(|v|+\left|v^{\prime}-v^{\prime \prime}\right|\right) D^{\prime} D^{\prime \prime}} \mathrm{M}_{2}^{\prime D^{\prime \prime}} \mathrm{M}_{2}^{\prime \prime D^{\prime}} & \left(\left|v^{\prime}\right|+\left|v^{\prime \prime}\right| \geq|v|+\left|v^{\prime}-v^{\prime \prime}\right|, \text { valid even for } v=0\right) \\
& \geq 2^{|v| D} 2^{D+\left|v^{\prime}-v^{\prime \prime}\right| D^{\prime} D^{\prime \prime}} \mathrm{M}_{2}^{\prime D^{\prime \prime}} \mathrm{M}_{2}^{\prime \prime D^{\prime}} & \left(D^{\prime} D^{\prime \prime} \geq D\right) \\
& =2^{|v| D} \mathrm{M}_{2}(E) & & \text { (Measure[2] Rules). }
\end{array}
$$

Line $5, E=\sqrt[p]{E^{\prime}}$.

$$
\begin{array}{rlrl}
\mathrm{M}(E) & =\mathrm{M}^{\prime} & & \text { (Measure Rules) } \\
& \geq 2^{\left|v^{\prime}\right| D^{\prime}} M_{2}^{\prime} & & (\text { by induction }) \\
& =2^{(p|v|+|m|) D^{\prime}} M_{2}^{\prime}\left(\left|v^{\prime}\right|=p|v|+|m|\right) \\
& =2^{|v| D} \mathrm{M}_{2}(E) & & \left(D=p D^{\prime}, \text { Measure[2] Rules }\right) .
\end{array}
$$

Note that $\left|v^{\prime}\right|=|v|+|m|$ holds because of rounding towards 0 .

Line $6, E=E^{\prime k}$.

$$
\begin{aligned}
\mathrm{M}(E) & =\mathrm{M}^{\prime k} \quad \text { (Measure Rules) } \\
& \geq 2^{\left|v^{\prime}\right| k D^{\prime}} M_{2}^{\prime k} \quad \text { (by induction) } \\
& =2^{|v| D} \mathrm{M}_{2}(E) \text { (Measure[2] Rules). }
\end{aligned}
$$

Line $7, E=\operatorname{Root}\left(F_{n}, \ldots, F_{0}\right)$. If we adopt the trivial rule where $v(E)=0$ then the inequality of this lemma is also trivial.

The main domination result is now easy to show:

THEOREM 7 Let $E$ be any expression involving the operators of Table 4. Assuming the trivial rule for the root operator, we have

$$
\beta(E) \leq \beta_{2}(E) .
$$

PROOF. We have

$$
\begin{array}{rll}
\beta(E) & =\frac{1}{\mathrm{M}(E)} & \\
& \leq \frac{1}{\mathrm{M}_{2}(E) 2^{|v(E)| D}} \quad & (\text { preceding Lemma }) \\
& \leq \frac{2^{v(E)}}{\mathrm{M}_{2}(E)} & (|v(E)| D+v(E) \geq 0) \\
& =\beta_{2}(E) . &
\end{array}
$$


Table 6

Relative effectiveness of 3 Root Bounds on CORE Test Suite

\begin{tabular}{|c||r|r|r|}
\hline & original BFMSS & BFMSS[2] & BFMSS[2,5] \\
\hline \hline BFMSS family & $55712 / 4016$ & $55726 / 14214$ & $55746 / 15277$ \\
\hline Li-Yap & $51669 / 33$ & $41531 / 19$ & $40472 / 3$ \\
\hline degree-measure & $4 / 4$ & $4 / 4$ & $0 / 0$ \\
\hline \hline Total number of expressions & 55749 & 55749 & 55749 \\
\hline
\end{tabular}

\section{Experimental Results}

The timings in this paper are based on runs on an Ultrasparc 10 machine with a $440 \mathrm{MHz}$ CPU. The software is Core Library Version 1.5+, which implements ${ }^{8}$ the Measure Bound, the Li-Yap Bound and a choice between the original BFMSS, the BFMSS[2], or the BFMSS[2,5] Bound.

To give empirical data on the relative effectiveness of these three bounding functions, we ran the Core Library Test Suite and counted the number of times that each bounding function is the best one. The results are shown in Table 6. Note that more than one bounding function may be best for an expression. So for each bounding function $\beta$, we give a pair $m / n$ of numbers where $m$ is the number of times that $\beta$ achieves the best bound, and $n$ is the number of times $\beta$ is the unique best bound. Thus $m \geq n$. The first column gives the result of a run with the original BFMSS Bound used, the second column gives the result of a run with the BFMSS[2] Bound used, and the third column gives the result of a run with the BFMSS[2,5] Bound used. We conclude from this table that the $(2,5)$-ary version of BFMSS, for all practical purposes, dominates the other two bounding functions in our test suite.

Experiment 1 involves the expression $E_{1}(x, y)$ given in the introduction. We assume that $E_{1}(x, y)$ does not share subexpressions. For example, we can reduce the degree from 16 to 8 by sharing, and the bit-bound function for BFMSS improves to $48 L+22$.

Experiment 2 involves the expression $E_{2}(x, y)=\frac{\sqrt{x}-\sqrt{y}}{x-y}-\frac{\sqrt{x}-\sqrt{y}}{x-y}$, an example from [3]. When $x, y$ are integers, the bit-bound from BFMSS and Li-Yap are

\footnotetext{
8 Version $1.5+$ refers to the modifications of the released Version 1.5 necessary to support the experiments of this paper. Our implementation of these bounds will generally return slightly worse bounds than the theory predicts because we maintain upper bounds on $\lg \mathrm{M}(E), \lg u_{2}(E)$, etc, instead of $\mathrm{M}(E), u_{2}(E)$, etc. The Core Library Test Suite is a set of about 30 sample programs that is distributed with the library.
} 
Table 7

Bitbound for dynamic programming determinant for random binary entries ( $L=$ 100)

\begin{tabular}{|l||l|l||l|l|}
\hline$n$ & $(n !) n L$ & BFMSS & $n L$ & BFMSS[2] \\
\hline \hline 2 & 400 & 164 & 200 & 101 \\
3 & 1800 & 657 & 300 & 169 \\
4 & 9600 & 2267 & 400 & 248 \\
5 & 60,000 & 10,468 & 500 & 326 \\
\hline
\end{tabular}

$6 L+64$ and $65 L+91$, respectively. But when $x, y$ are $L$-bit binary numbers, the bit-bound of BFMSS[2] is $7.5 L+11$. When we substitute various machine double values, we obtain bit-bounds whose ranges are: 1643-1707 (BFMSS), 323-331 (BFMSS[2]). Running these 1000 times gives timings of 36 seconds (BFMSS) and 22.8 seconds (BFMSS[2]). Although there is an improvement, it is not of the order of magnitude one might expect from bit-bound ranges; this seems to be an implementation-induced effect.

Determinants. Experiment 3 involves the determinant example in the introduction. Let $A$ be a $n \times n$ matrix whose entries are $L$-bit binary rationals. By definition, the entries have the form $n 2^{-k}$ where $0 \leq n<2^{L}$ and $0 \leq k \leq L$. There are two special cases that we consider:

(1) If $n \geq 2^{L-1}$, we say the $L$-bit binary rational is strict. All the numbers in $A$ are strict in our experiment.

(2) If $k=L$, then we say the $L$-bit binary rational is normal.

We noted that if $E_{0}$ is the co-factor expansion of matrix $A$, then the BFMSS bound gives $-\lg \beta^{\text {bfmss }}\left(E_{0}\right) \leq(n !) n L$, while the binary BFMSS bound gives $-\lg \beta_{2}^{\text {bfmss }}(E) \leq n L$. If $E^{\prime}$ is the dynamic programming implementation of the determinant of $A$, then $\beta^{\text {bfmss }}\left(E^{\prime}\right)$ may be strictly greater than $\beta(E)$. For instance, if $a, b, c$ are $L$-bit binary numbers then $\beta^{\text {bfmss }}(a(b+c))=3 L$ while $\beta^{\text {bfmss }}(a b+a c)=4 L$. On the other hand, $\beta_{2}^{\text {bfmss }}(a(b+c))=\beta_{2}^{\text {bfmss }}(a b+a c)$. Table 7 compares the root bit bounds of BFMSS and the binary version on random matrices whose entries are 100-bit binary rationals. These empirical bounds are (as expected) better than the worst case estimate. If we use normal 100-bit binary rationals, then Table 8 gives the same comparison when those entries are normalized 100-bit binary rationals. Our implementation of the $\beta_{2}^{\text {bfmss }}$ bound practically matches the theoretical upper bound of $n L$.

We next compare timing for BFMSS, BFMSS[2] and BFMSS[2,5]. Despite the wide gap in the root bounds, the timings are not expected to be different for random matrices. That is because a random determinant is unlikely to be zero and so the floating point filter will be in effect. Instead, we convert the above data into degenerate matrices, just by making the last row a duplicate of the previous row. Surprisingly, there was no detectable difference in tim- 
Table 8

Bitbound for dynamic programming determinant for random normal binary entries $(L=100)$

\begin{tabular}{|l||l|l||l|l|}
\hline$n$ & $(n !) n L$ & BFMSS & $n L$ & BFMSS[2] \\
\hline \hline 2 & 400 & 400 & 200 & 200 \\
3 & 1800 & 1497 & 300 & 300 \\
4 & 9600 & 6364 & 400 & 400 \\
5 & 60,000 & 32,282 & 500 & 499 \\
\hline
\end{tabular}

Table 9

Dynamic programming determinant for degenerate strict matrices with 50-digit decimal rationals

\begin{tabular}{|l||l|l||l|l||l|l|}
\hline \multicolumn{1}{|c||}{$n$} & \multicolumn{2}{c||}{ BFMSS } & \multicolumn{2}{c||}{ BFMSS[2] } & \multicolumn{2}{c|}{ BFMSS[2,5] } \\
\hline & time & bitbd & time & bitbd & time & bitbd \\
\hline \hline 2 & 4.4 & 352 & 4.3 & 300 & 4.4 & 175 \\
\hline 3 & 15.2 & 648 & 13.9 & 538 & 14.1 & 236 \\
\hline 4 & 57.5 & 2624 & 58 & 1984 & 35 & 339 \\
\hline 5 & 568 & 15,427 & 572 & 12,202 & 107 & 444 \\
\hline
\end{tabular}

ing between BFMSS and BFMSS[2]. This could be explained by two effects. The first is that the internal representation of the numbers are in binary, and even when the root bound asks for many bits of precision, our implementation of BigFloat ensures that trailing zeroes are omitted. The second is that the precision of the internal approximation is increased each time by a factor of two until the bitbound is reached. This gives a "step effect" in the function expressing the running time in terms of the bitbound. The improvement of the BFMSS[2] bitbound over BFMSS must be greater than a factor of 2 in order to guarantee observability. In fact, a slight slowdown is sometimes detectable because of the extra steps to maintain the 2-ary version of BFMSS. To overcome the first effect, we avoid inputs that are purely binary; our next set of experiments use decimal rationals. The second effect, unfortunately, persists. We use random (degenerate) matrices whose entries are strict 50-digit decimal rationals. Table 9 compares the speed of BFMSS, BFMSS[2] and BFMSS[2,5]. The timings are for 10,000 evaluations of each determinant.

\section{Open Problems and Future Work}

This paper introduced the factoring technique into constructive root bounds, and demonstrated its effectiveness. In general, the problem of constructive root 
bounds will become more important as EGC techniques and such algorithms become more widely used. The trade-offs between effectiveness (i.e., small root-bit bounds) and efficiency (i.e., low computational complexity) is not understood. Between the extremes of simple recursive rules (that constitute the bulk of current bounds) and (say) computing minimal polynomials, we would like to see methods with intermediate computational complexity. Our factoring method can be seen as one step in this direction. We list some open problems and future work:

- Our $k$-ary method can be generalized to maintain arbitrary rational factors, in addition to $k$-ary factors. (e.g., transform $E$ to $q 2^{v} E_{2}$ where $v \in \mathbb{Z}$, $q \in \mathbb{Q}$ ). The benefit of the rational factors is less predictable, and hence experimentation is called for.

- Current constructive root bound techniques are mostly static in nature. More dynamic root bound techniques should be exploited. An idea of Sekigawa [14] can be pursued. Sekigawa proposed some methods in the case of the measure bound, but they do not seem to have been implemented. We could combine with the most significant bit (MSB) bound that is maintained in the Core Library [7].

- It is clear that the $k$-ary method can also be applied to the Li-Yap Bound.

- The general treatment of the diamond operators under the Measure Bound is a subject for further research.

- The incorporation of the Sekigawa improvements into the current Measure[2] Rules is immediate if there is no division. It is possible to give rules that incorporate these improvements for division, but it is unclear how to ensure that the binary bound dominates the original bound.

Postscript. Recently, Susanne Schmitt [13] has extended the techniques of this paper for the diamond operator $\diamond\left(F_{n}, \ldots, F_{0}\right)$, and validated the theoretical improvements by experiments.

\section{References}

[1] C. Burnikel, R. Fleischer, K. Mehlhorn, and S. Schirra. Exact geometric computation made easy. In Proc. 15th ACM Symp. Comp. Geom., pp 341450, 1999 .

[2] C. Burnikel, R. Fleischer, K. Mehlhorn, and S. Schirra. A strong and easily computable separation bound for arithmetic expressions involving radicals. Algorithmica, 27:87-99, 2000.

[3] C. Burnikel, S. Funke, K. Mehlhorn, S. Schirra, and S. Schmitt. A separation bound for real algebraic expressions. In 9th ESA, volume 2161 of Lecture Notes in Computer Science, pp 254-265. Springer, 2001. 
[4] Core Library homepage, Since 1999. Software downloads, documentation and links: http://cs.nyu.edu/exact/core/.

[5] V. Karamcheti, C. Li, I. Pechtchanski, and C. Yap. A Core library for robust numerical and geometric libraries. In Proc. 15th ACM Symp. Comp. Geom., pp 351-359, 1999.

[6] LEDA Homepage, Since 1994. URL http://www.mpi-sb.mpg.de/LEDA/. Library of Efficient Data Structures and Algorithms (LEDA) Project. From the Max Planck Institute of Computer Science.

[7] C. Li. Exact Geometric Computation: Theory and Applications. Ph.D. thesis, New York University, Department of Computer Science, Courant Institute, Jan. 2001. Download from http://cs.nyu.edu/exact/doc/.

[8] C. Li and C. Yap. A new constructive root bound for algebraic expressions. In Proc. 12th ACM-SIAM Symp. on Discrete Algorithms, pp 496-505, Jan. 2001.

[9] M. Marden. The Geometry of Zeros of a Polynomial in a Complex Variable. Math. Surveys. American Math. Soc., New York, 1949.

[10] M. Mignotte. Identification of algebraic numbers. J. of Algorithms, 3:197-204, 1982.

[11] G.-C. Rota. Finite Operator Calculus. Academic Press, Inc, 1975.

[12] E. R. Scheinerman. When close enough is close enough. Amer. Math. Monthly, 107:489-499, 2000.

[13] S. Schmitt. Improved separation bounds for the diamond operator. Technical Report ECG-TR-363108-01, ECG Project (Effective Computational Geometry for Curves and Surfaces). INRIA Sophia-Antipolis, 2004. (13 pages) ftp-sop.inria.fr/prisme/ECG/Reports/Month36/ECG-TR-363110-01.ps.gz.

[14] H. Sekigawa. Using interval computation with the Mahler measure for zero determination of algebraic numbers. Josai Information Sciences Researches, 9(1):83-99, 1998.

[15] C. K. Yap. Fundamental Problems of Algorithmic Algebra. Oxford University Press, 2000.

[16] C. K. Yap. On guaranteed accuracy computation. In F. Chen and D. Wang, editors, Geometric Computation. World Scientific Publishing Co., Singapore, 2004 .

[17] C. K. Yap and T. Dubé. The exact computation paradigm. In D.-Z. Du and F. K. Hwang, editors, Computing in Euclidean Geometry, pp 452-486. World Scientific Press, Singapore, 2nd edition, 1995. 